\title{
Analyse de genre : la critique de cinéma dans la presse quotidienne britannique
}

Dorothée Baud

\section{CpenEdition}

\section{Journals}

Édition électronique

URL : http://journals.openedition.org/asp/1282

DOI : 10.4000/asp. 1282

ISBN : 978-2-8218-0392-3

ISSN : 2108-6354

Éditeur

Groupe d'étude et de recherche en anglais de spécialité

Édition imprimée

Date de publication : 1 mai 2003

Pagination : $37-45$

ISSN : 1246-8185

Référence électronique

Dorothée Baud, «Analyse de genre : la critique de cinéma dans la presse quotidienne britannique », ASp [En ligne], 39-40 | 2003, mis en ligne le 04 mai 2010, consulté le 19 avril 2019. URL : http:// journals.openedition.org/asp/1282 ; DOI : 10.4000/asp.1282

Ce document a été généré automatiquement le 19 avril 2019

Tous droits réservés 


\title{
Analyse de genre : la critique de cinéma dans la presse quotidienne britannique
}

\author{
Dorothée Baud
}

\section{Introduction}

1 «Is there [...] a limited finite set of genres or is the world of discourse characterized by infinite textual variety?» (McCarthy \& Carter 1994 : 32). Si la question est soulevée par ces deux auteurs, c'est bien parce que définir genres et sous-genres se révèle être une tâche bien plus difficile que ce que l'intuition première pourrait laisser penser. Face à la diversité des discours particuliers, on ne peut définir un genre qu'en adoptant une démarche assez souple pour intégrer des textes particuliers qui présentent, au-delà de leurs caractéristiques communes, des différences plus ou moins importantes. La notion de Generic Structure Potential (GSP), introduite par Hasan (1984), est à cet égard d'une grande utilité. Le GSP est ainsi défini : «an abstract category [...] descriptive of the total range of textual structures available within a genre $G[. .$.$] designed to highlight the variant and invariant$ proprieties of textual structures within the limit of genre » (cité par Cockcroft $1992: 101)$ ). Mais pour pouvoir analyser ces éléments obligatoires et optionnels, il faut d'abord postuler l'existence d'un genre, et donc établir des critères pour envisager un ensemble de textes comme appartenant à une même catégorie. Comme le remarque Cook, en effet,

$[t]$ here are [...] many different ways of categorizing discourse types: by situation, by

function, by participants, by text, by substance, or by a combination of these

factors. Consequently, a given discourse may be several types at once. $(1992: 4)$

Il semble cependant que les aspects contextuels constituent de bons critères pour procéder à une première classification des types de discours. Biber et al. ont ainsi choisi d'établir des distinctions génériques (ils emploient le terme anglais register pour désigner le genre) fondées sur des critères non linguistiques: «Register distinctions are defined in non-linguistic terms, with respect to situational characteristics such as mode, interactiveness, domain, communicative purpose, and topic » (1999: 15). Dans le cas des critiques de cinéma, l'environnement contextuel des articles suggère leur appartenance à un genre: ils 
apparaissent en effet dans une section du journal généralement intitulée Reviews, dans laquelle une page spéciale est dédiée aux Film reviews quand il y a plusieurs types de critiques (livres, pièces de théâtre, expositions, etc.). On peut donc faire l'hypothèse qu'il existe un genre "critique de cinéma ", qui serait un sous-genre du type de discours qu'on pourrait appeler "article de critique ", lui-même appartenant au genre plus large du discours journalistique. Cette hypothèse doit cependant être confirmée : l'appellation commune à ces différents articles reflète-t-elle une réelle unité, tant au niveau de la forme que du contenu? Il convient pour répondre à cette question d'entreprendre une analyse de genre proche de celle que Swales définit comme "[a] system of analysis that is able to reveal something of the patterns of organization of a genre and the language used to express those patterns » (1990: 50). Dans la perspective d'analyse de discours qui est celle de cette étude, ces caractéristiques textuelles sont à mettre en relation avec le contexte, pris dans un sens large.

D'un point de vue méthodologique, le point de départ de cette étude a été de rassembler, une semaine donnée, tous les journaux quotidiens nationaux britanniques, ainsi que leur version dominicale, et d'examiner les différentes critiques consacrées à un même film. Dans tous les journaux de la semaine du 20 novembre 2000, le film faisant l'objet de l'article le plus long était Charlie's Angels. Le corpus se compose donc d'une quinzaine de critiques de ce film. Il apparaît très vite à la lecture de ces textes que le journaliste a une grande liberté par rapport au style et au contenu de son article. Ainsi, certains critiques n'hésitent pas à raconter des anecdotes, ou bien à faire des digressions sur divers sujets dans leur article. La critique du Daily Telegraph commence par exemple par un récit assez long qui introduit des remarques sur la tendance du cinéma à « recycler » d'anciennes fictions télévisuelles :

Not long ago I experienced the most embarrassing overheard conversation of my life. I was in one of those places in Soho when they charge you four million quid for a bottle of beer [...]. The producer and actor were making a pitch. They furrowed their brows and clawed the air with great feeling. [...] [T]hey were discussing Mr Benn, the 1970s children's cartoon which they thought would make the greatest movie ever. "It's about a spiritual journey," said the producer, "a modern journey into fantasy and redemption." "It's about finding truth," said the actor.

4 D'autres journalistes évoquent dans leur article la vie privée des actrices (Sun, Daily Express, mais aussi The Times), ébauchent une analyse sociologique (par exemple sur la violence perpétrée par les femmes dans le Daily Mail), ou plus psychologisante (« we are all, to various degrees, retrophiliacs - that is, people with a passionate love for the dead television shows of their youth", Sunday Times). Si la critique d'un même film peut donner lieu à des développements aussi divers, est-il encore légitime de parler de genre?

En dépit de cette indéniable variété, un certain nombre de points communs entre les articles du corpus apparait d'emblée, dont certains sont assez prévisibles. Toutes les critiques contiennent à la fois des informations sur le film et l'expression d'un jugement sur celui-ci, ce qui est à mettre en relation avec les deux fonctions premières de la critique, qui sont d'une part de présenter un nouveau film, et d'autre part d'en évaluer la valeur pour convaincre le lecteur d'aller le voir ou non. C'est pourquoi dans toute critique figurent au moins le nom de quelques acteurs et/ou du réalisateur, le genre du film, quelques éléments de l'histoire, ainsi qu'un commentaire pris en charge par le journaliste. Pour ne donner que deux exemples de ce dernier aspect, citons le critique du 
Times, qui juge le film « unexpectedly loveable», tandis que celui du Mirror affirme: "The script is good and the supporting cast [...] is terrific ». Les critiques partagent également des points communs avec les autres articles de journaux, notamment au niveau syntaxique, dans la mesure où la concision y est de mise. On trouve donc de nombreux syntagmes nominaux complexes, très souvent avec des propositions relatives. Un exemple tiré du Sunday Express montre comment les journalistes peuvent donner un maximum d'informations en un minimum de mots :

The Angels are hired via their invisible boss Charlie, to rescue the kidnapped founder of Knox technologies (Sam Rockwell) who has developed a voice identification program which can produce a DNA-accurate profile of someone's voice.

6 Cet article ne s'attardera pas sur ces éléments, mais s'intéressera à d'autres points communs qui présentent un intérêt plus grand dans le cadre de la caractérisation du genre : ceux, tout d'abord, qui découlent du caractère persuasif de la critique, puis les aspects divertissants et ludiques de ces articles. Enfin, nous évoquerons brièvement les applications pédagogiques possibles de l'étude de ce genre.

\section{Un discours de type persuasif}

7 Pour expliquer cette dimension cruciale du genre de la critique en général, il convient d'examiner le contexte de ce type de discours, et plus particulièrement les participants qu'il implique. Le critique a vu un film que le lecteur, lui, n'a pas vu: en effet, la critique est généralement publiée le jour de la sortie des films en Grande-Bretagne, c'est-à-dire le vendredi. Une des fonctions principales de ce type d'article est donc de guider le lecteur dans son choix de film. La situation est par conséquent différente du cas des rubriques consacrées par exemple aux émissions télévisées qui ont déjà été diffusées, et que le lecteur a donc eu la possibilité de voir.

De nombreuses caractéristiques du genre découlent de la fonction persuasive de ce discours. Pour rallier le lecteur à son opinion, le journaliste utilise des procédés classiques. Ainsi, les verbes par lesquels il exprime un jugement ne sont quasiment jamais modalisés. Les exemples sont nombreux dans les articles étudiés : « a script which is as lazy as it is moronic » (Daily Mail), « the movie's pretty good " (Mail on Sunday), "[t]he movie passes the time" (Observer), etc. En utilisant dans les énoncés évaluatifs le présent simple, le critique présente ainsi ses opinions de façon péremptoire. Car, comme le souligne Lyons, " categorical assertions express the strongest degree of speaker commitment. [...] You are right is actually epistemically stronger than the modalized You must be right " (cité par Simpson 1993 : 49-50). Le corpus ne contient en tout que deux occurrences de modalisation épistémique utilisée pour nuancer un jugement, dont l'un se trouve dans le Mirror : « it may well be the first genuinely modern $21^{\text {st }}$ century blockbuster ». La modalité peut être utilisée au contraire pour renforcer des assertions, comme c'est le cas dans le même article : «It's so upfront and honest you can't possibly object ».

9 Dans sa perspective argumentative, le critique a également recours à des figures de rhétorique. Les métaphores sont ainsi fréquemment utilisées pour leurs connotations négatives, comme dans l'article du Daily Telegraph: « a movie in which the obvious is a slave to the irrelevant ». Le même critique file une autre métaphore pour railler le film : « they set 
out to karate-chop their way through the male world [...] they mainly have to fight their way through the lamest script of the year so far ».

L'utilisation du pronom personnel you participe également de la dimension persuasive dans certaines critiques. Il est en effet présent dans quasiment tous les articles, et permet de créer une sorte de complicité avec le lecteur. Mais le référent de ce pronom est parfois quelque peu ambigu, à dessein semble-t-il. En effet le lecteur peut se demander s'il s'agit d'un you générique ou s'il fait référence au critique. Dans l'article du Daily Telegraph, par exemple, certaines occurrences du pronom font clairement référence au lecteur («before you know it, your tiny daughters will be rapid-punching the dog»), tandis que d'autres renvoient plus probablement au critique, même si elles peuvent recevoir une interprétation générique: "you can't help thinking that it must be like the bad old days for Barrymore ». La référence est parfois encore plus équivoque, comme dans «it takes you three nanoseconds to work out who the baddy is » (Daily Telegraph). L'utilisation de ce pronom permet ainsi d'inclure subrepticement le lecteur dans l'expression du jugement du journaliste.

11 Mais l'aspect le plus important pour l'efficacité de la persuasion concerne de façon plus générale l'image que le critique cherche à donner de lui-même dans son article. Pour analyser cet aspect, il convient de faire référence au concept d'Auteur Modèle introduit par Eco, qu'il décrit ainsi : « [L]e lecteur empirique, en tant que sujet concret des actes de coopération, doit [...] se dessiner une hypothèse d'Auteur en la déduisant des données de stratégie textuelle» (1985: 77). Cette image de l'auteur, à bien distinguer de l'auteur empirique, de la personne de chair et de sang, est créée en grande partie en fonction du Lecteur Modèle, qui est l'hypothèse de lecteur auquel se réfère l'auteur quand il écrit : «Pour organiser sa stratégie textuelle, un auteur doit se référer à une série de compétences [...] qui confèrent un contenu aux expressions qu'il emploie. Il doit assumer que l'ensemble des compétences auquel il se réfère est le même que celui auquel se réfère son lecteur. C'est pourquoi il prévoira un Lecteur Modèle capable de coopérer à l'actualisation textuelle » $(1985: 67-68)$.

Mais, comme le précise Eco, "prévoir son Lecteur Modèle ne signifie pas uniquement 'espérer' qu'il existe, cela signifie aussi agir sur le texte de façon à le construire » (1985 : 69). L'étude du corpus révèle une grande diversité des Auteurs Modèles en fonction des différents journaux pour lesquels ils écrivent, ce qui n'a rien de surprenant compte tenu de la variété de leur public cible. Cependant, on retrouve une constante dans quasiment tous les articles: l'image que donne le critique de lui-même est double. D'une part, il se présente comme un expert, un spécialiste du cinéma, qui a vu de nombreux films et qui a un certain savoir. Il fait donc souvent référence à d'autres films, généralement dans un but comparatif : la critique de Charlie's Angels donne lieu à des allusions aussi bien aux films de James Bond qu'à The Matrix ou Mission: Impossible, mais aussi à des films moins connus, surtout dans les journaux de qualité : Play it Again, Sam (Independent on Sunday), Blood and Wine (Sunday Times), The Saint (Independent), Modesty Blaise et Barbarella (Daily Express). Le critique est également très informé sur le monde du cinéma, sur les acteurs, les réalisateurs, les producteurs, etc. On peut alors se demander si cet « expert » utilise un vocabulaire spécialisé. Il apparaît en fait que, si les critiques contiennent bien évidemment des termes appartenant au champ lexical du cinéma, le vocabulaire employé n'est en fait pas particulièrement technique : on trouve généralement des termes comme sequence, scene, big-screen. Le Times fait dans une certaine mesure exception, car le critique mentionne quelques aspects techniques du film, ce qui le conduit par exemple à utiliser 
l'expression split screen. Le critique légitime donc ses opinions en se présentant comme un spécialiste dans son domaine. Mais il cherche d'autre part à apparaitre proche du spectateur, ou plutôt du spectateur potentiel qu'est son lecteur, plus exactement son «Lecteur Modèle ». Ceci est particulièrement flagrant au niveau des nombreuses allusions culturelles que l'on trouve dans les critiques, qui sont très révélatrices de l'image que le journaliste veut donner de lui. Ainsi, certains articles contiennent des références qui renvoient à une culture que l'on peut qualifier de populaire ou, à l'instar des anglophones, de low-brow: allusions à l'animateur de radio Bruno Brookes (Mirror), à la chanteuse Geri Halliwell (Mail on Sunday), ou encore au film Belles of St Trinian's dans l'article du Mail on Sunday (" giggle like the fifth form at St Trinian's ", " the Alastair Sim role »). D'autres critiques empruntent leurs références à une sphère culturelle différente ( high-brow culture), comme c'est le cas de celui du Daily Telegraph, qui cite les philosophes Bertrand Russel et Ludwig Wittgenstein, et reprend de façon parodique, dans « Murray, with the face that launched a thousand fists ", une célèbre citation de la pièce de théatre The Tragical History of Dr Faustus de Marlowe, dans laquelle il est dit d'Hélène de Troie qu'elle est « the face that launched a thousand ships ». Ainsi, en distillant dans son article des références qu'il pense partager avec son lecteur, le critique cherche à créer une complicité avec lui, et permettre une identification propice à l'efficacité de la persuasion. Ce jeu de proximité et de distance du critique par rapport à son destinataire est également manifeste dans le mélange de niveaux de langue au sein des critiques. En effet, on peut trouver dans un même article, par exemple celui du Sunday Times, des termes comme butt (qui apparaît cinq fois), chicks (trois occurrences), foxy, kooky, ou sass, mais également retrophiliacs ou eclecticism.

13 Cette double dimension a certes une fonction persuasive en tant qu'elle participe de l'élaboration de l'image du critique. Mais c'est aussi un aspect d'une caractéristique que l'on trouve dans tous les articles du corpus sans exception : une tendance à jouer avec les mots, avec la langue.

\section{Humour, code play et creative writing}

14 L'humour semble être une caractéristique invariante du genre critique de cinéma, même s'il est plus ou moins présent selon les journaux, et qu'il apparaît sous différentes formes. Les procédés humoristiques sont en effet divers, mais ce sont souvent les comparaisons qui sont utilisées pour leur pouvoir évocateur potentiellement comique : ainsi, dans l'article du Daily Mail, le critique compare Cameron Diaz à un ornythorinque («Diaz [...] starting to look like an emaciated duck-billed platypus »). Ces comparaisons font souvent appel à des allusions culturelles à des fins humoristiques : «Cameron Diaz wears a desperate, fixed grin reminiscent of Fanny Cradock dismembering a haddock » (Daily Mail), ou encore, à propos de Drew Barrymore, "her fight scenes have all the grace and athleticism of John Prescott attempting the title role in Billy Elliot » (Daily Mail). D'autres allusions humoristiques sont des reprises parodiques d'autres types de discours: dans le Daily Star, par exemple, l'expression « whiter-than-white teeth » rappelle certains slogans publicitaires.

L'humour passe aussi et surtout dans les critiques par les jeux de mots, à la thématique parfois réccurrente dans certains articles, comme dans celui du Sunday Times : «Diaz, who has a great future behind her ", "Not since Jennifer Lopez appeared in Blood and Wine has a star's bottom been on everybody's lips ». Les jeux de mots sont par ailleurs systématiquement présents dans les titres des articles (« Heavenly Angels » (Sun), « Why these Angels are proper 
Charlies» (Daily Mail)), ainsi que dans les légendes des photos du film publiées avec la critique : "Tat's All Folks» (The Independent). Ces jeux avec la langue impliquent souvent, eux aussi, des allusions culturelles : c'est le cas du titre «Top of the Chops " (Guardian), ou de celui de l'article dans The Independent, « Girls just want to have stunts », qui fait référence à la chanson de Cyndi Lauper "Girls just want to have fun ".

De manière plus générale, les critiques semblent éprouver une véritable délectation à manier la langue et à créer un style personnel. Ainsi, de nombreux journalistes ont recours à des procédés de composition lexicale, à la fois dans un but de concision et dans une perspective créative. La plupart des ces composés apparaissent en position adjectivale, tels «[t]he take-no-bullshit babes » (Daily Mail) ou " must-see-twice movie » (Sun). Le record de la longueur dans le corpus revient au critique du Daily Express, qui qualifie l'actrice Drew Barrymore de "child-star-turned-wild-child-junkie dumper» (Daily Express). On trouve également dans plusieurs articles des jeux sur les sonorités, avec des allitérations telles que " go gooey and giggle » (Mail on Sunday), "kooky high-kicking blonde » ( Sunday Times), ou encore «kick-boxing whizz is a bikini waxer " (Mirror). Les deux exemples de comparaisons humoristiques citées plus haut contiennent également des rimes intérieures : «Cradock» et « haddock » d'une part, et « Prescott » et « Elliot » d'autre part. En outre, certains critiques semblent apprécier les mots peu usités ou quelque peu recherchés comme «lederhosen » ou «pustulent» (Daily Express), tandis que d'autres se plaisent à utiliser à l'écrit des tournures orales, comme c'est le cas en particulier dans l'article du Sun avec « blah, blah, blah » ou encore «sooooo cool».

Comment peut-on expliquer cette dimension divertissante, la présence si systématique de procédés qui relèvent du code play? Il faut tout d'abord bien souligner que la page consacrée aux critiques se trouve en général dans une section séparée ou à la fin du journal. C'est pourquoi, plus encore qu'un autre article, la critique doit attirer l'attention du lecteur pour être lue. De plus, comme elle se trouve dans un journal généraliste mais que son sujet est assez spécialisé, elle a un lectorat potentiellement restreint de cinéphiles ou en tout cas de gens qui vont régulièrement au cinéma. Pour avoir une chance d'être lue par le maximum de lecteurs, la critique de cinéma doit donc avoir un autre intérêt, s'affranchir de ses fonctions premières (informer et évaluer). Ainsi Keeble souligne-t-il que " [t] he review must [...] exist as a piece of writing in its own right. It must entice the reader through the quality and colour of its prose. It must entertain, though different papers have different conceptions of what entertainement means » (1994:301).

Bywater \& Sobchack vont même jusqu'à comparer ce type d'article à la littérature, ce que suggéraient les remarques faites sur tous les procédés de jeux avec la langue et notamment les allitérations : "[reviewers] are writers as well as film critics and it shows in their work. Indeed their reviewing is a kind of literature - the witty and personal critical essay which can be read for its own sake» $(1989: 16)$.

retrouve cette idée dans la préface d'une anthologie d'articles de la critique américaine Pauline Kael. William Shawn y décrit en effet « the wit and energy and clarity of her prose » et conclut en écrivant : " what she is primarily is a writer; one reads her for the sheer pleasure her writing affords » (1993:v).

20 La critique de cinéma apparaît donc comme un genre à part entière, ou peut-être plutôt (l'hypothèse reste à explorer) un sous-genre de l'article de critique. Ce type d'analyse peut mener à des applications pédagogiques dans la mesure où les caractéristiques génériques qui ont été mises en évidence présentent plus d'un intérêt pour l'enseignement de l'anglais. 


\section{L'utilisation de critiques de cinéma en classe de langue}

21 Les critiques de film peuvent en effet constituer un outil intéressant dans le cadre de l'apprentissage de la langue, avec des apprenants ayant déjà un bon niveau de compréhension et d'expression. L'expérience a été menée à l'École normale supérieure de Cachan, avec deux groupes de première année (niveau licence), respectivement en magistère de physique et d'informatique. Ces étudiants, en petits groupes et motivés pour perfectionner leur anglais, reçoivent à l'ENS des cours combinant langue de spécialité et anglais général. Les critiques de cinéma peuvent, dans cette perspective plus généraliste, être utilisées comme supports de plusieurs types d'exercice.

Dans un premier temps, plusieurs articles peuvent être étudiés en classe, et donner lieu à un travail de compréhension et d'expression orale. En effet, certaines critiques contiennent, comme nous l'avons vu, un vocabulaire varié, et la diversité des niveaux de langue permet de sensibiliser les apprenants aux nuances de registre. Les critiques de Charlie's Angels offrent ainsi un échantillon varié de termes renvoyant à un même champ sémantique mais ne s'employant pas dans les mêmes conditions : «butt», "backside », " rear end", "bottom», ou "tushes», pour n'en citer que quelques-uns. Les allusions culturelles peuvent également être décodées, par les apprenants eux-mêmes ou par l'enseignant. Ainsi, certains étudiants de l'ENS savent ce à quoi l'adjectif « Blofeldian » fait référence dans le syntagme nominal « the villain's Blofeldian complex» (Mail on Sunday), car Blofeld est un personnage qui apparaît dans plusieurs films de James Bond. En revanche, d'autres références culturelles sont plus difficiles à décoder pour un Français, par exemple dans l'allusion aux Carry On comedies ( the kind of smutty jokes beloved of Carry On comedies » Daily Star), ou à « Farrah [Fawcett]'s Bride of Wildenstein presence» (Guardian). Après le travail de compréhension proprement dit, les étudiants sont amenés, à l'oral, à trouver les points communs entre les différents articles, afin de dégager les caractéristiques du genre. On peut par là même les faire réfléchir sur la notion même de genre, et introduire certains concepts théoriques relatifs aux problématiques génériques si ces aspects sont susceptibles de les intéresser. La mise en lumière des différences entre les critiques permet en outre de faire une mise au point civilisationnelle sur la presse britannique.

La deuxième partie de ce travail peut se centrer sur la production écrite d'une critique, sur un film choisi par l'enseignant ou par l'apprenant. L'exercice nécessite une prise en compte de ce qui a été dit sur les critiques étudiées, afin de respecter les conventions du genre. La rédaction d'une critique implique donc non seulement les compétences classiques de l'expression écrite mais aussi une capacité à manier la langue pour produire jeux de mots et effets rhétoriques. L'apprenant est également invité, dans le cadre de cet exercice, à tenter de personnaliser son style et à forger à travers son écriture l'image qu'il veut donner de lui-même, pour être le plus persuasif possible. L'inventivité requise par ce type d'exercice concerne également l'aspect visuel de la critique : la présentation se doit d'être semblable à celle d'un journal (titres, sous-titres, colonnes) mais peut être agrémentée de photographies du film, de citations mises en relief, etc. 


\section{Conclusion}

24 articles du corpus, afin de dégager les caractéristiques du genre. Il ne faut toutefois pas minimiser la grande diversité au sein de ce corpus, car, si certains invariants apparaissent dans tous les articles, les différences sont aussi très importantes d'un journal à l'autre. Cette variété est révélatrice des différents types de lectorat que les quotidiens privilégient. On note ainsi un contraste très marqué entre la complexité syntaxique de certains articles et les phrases de la critique du Sun, qui sont majoritairement des propositions indépendantes sans lien entre elles, plusieurs commençant par "it's », telles "It's brash, sexy, scintillating", "It is ironic and cheeky», "it's like the Matrix meets Austin Powers with mega-babes ». Cependant, l'analyse du corpus montre que la distinction entre les tabloïdes et les journaux de qualité n'apparaît pas toujours de façon évidente. Ainsi, par rapport au film, les opinions sont diverses et ne dépendent pas du type de journal. Certes, la critique de Charlie's Angels dans le Sun est dithyrambique, à l'inverse de celle publiée dans The Independent. Toutefois, le critique du Times juge le film favorablement, alors que celui du Daily Mail conclut son article par : «Compared to this, Ghostbusters was a masterpiece ». De même, les allusions au physique des actrices ne se trouvent pas que dans les quotidiens populaires. Sur un plan plus stylistique, les contrastes entre les différents articles ne sont pas toujours ceux que l'on pourrait imaginer. C'est en effet dans le Mirror que se trouvent les termes "embark» ou "protracted ", alors que "flick», "phoney ", " cheesy » apparaissent dans l'article du Daily Telegraph. Une des phrases les plus complexes syntaxiquement se trouve dans le Mail on Sunday :

I don't know what I dreaded more about this picture: the feeble idea of turning a cheesy Seventies television series into a feature film, or the worldwide torture that was bound to be inflicted on the century's most overused word, 'ironic', by earnest youngers who actually believe there was an entity (beyond the imagination of colour supplement fashion pages) called 'the Seventies'.

Ainsi, il semble bien que si le contexte, et en particulier le type de journal, a une influence importante sur les caractéristiques de la critique, elle n'est toutefois pas déterminante. De même, si l'appartenance au genre "critique de cinéma » a des conséquences discursives indéniables, elle n'induit pas des contraintes aussi strictes que d'autres types de discours, ce qui explique la variété qui existe au sein de ce genre.

\section{BIBLIOGRAPHIE}

\section{Références du corpus}

Billson, Anne. « Bubblegum Vabes ». The Sunday Telegraph, 26 novembre 2000.

Bradshaw, Peter. « Top of the Chops ». The Guardian, 24 novembre 2000. 
Falk, Quentin. « Thank Heaven for the Dazzling Diaz ». The Sunday Mirror, 26 novembre 2000.

Faulks, Sebastian. « Bare Cheek that Makes the Angels so Heavenly ». The Mail on Sunday, 26 novembre 2000.

Fisher, Nick. « Heavenly Angels ». The Sun, 25 novembre 2000.

Fitzherbert, Henry. « Leaping, Kicking, Gravity-defying Sleuths Put Men Firmly in their Place. » The Sunday Express, 26 novembre 2000.

Frank, Alan. « Fallen Angels. » The Daily Star, 24 novembre 2000.

French, Philip. « Charlie and the Hot-Chick Factory ». The Observer, 26 novembre 2000.

Gilbey, Ryan. « Girl Power ». The Daily Express, 24 novembre 2000.

Landesman, Cosmo. « They're no Angels ». The Sunday Times, 26 novembre 2000.

Mars-Jones, Adam. « Big Girls, and One in a Blouse ». The Times, 23 novembre 2000.

O’Hagan, Andrew. « Three Girls Fight the Script ». The Daily Telegraph, 24 novembre 2000.

Quinn, Anthony. « Girls Just Want to Have Stunts ». The Independent, 24 novembre 2000.

Quirke, Antonia. « Pushing Charlie Makes the Money-men Happy ». The Independent on Sunday, 26 novembre 2000.

Ross, Jonathan. « Angels Delight ». The Mirror, 24 novembre 2000.

Tookey, Christopher. « Why these Angels are Proper Charlies ». The Daily Mail, 24 novembre 2000.

Whitworth, Melissa. «Film of the week : Charlie's Angels ». Play (The Times), 25 novembre- ( $^{\mathrm{er}}$ décembre 2000.

\section{Références}

Biber, Douglas et al. 1999. Longman Grammar of Spoken and Written English. Harlow : Longman. Bywater, Tim \& Thomas Sobchack. 1989. An Introduction to Film Criticism: Major Critical Approaches to Narrative Films. New York : Longman.

Cockcroft, Robert \& Susan. 1992. Persuading People. An Introduction to Rhetoric. Basingstoke : Macmillan.

Cook, Guy. 1992. The Discourse of Advertising. Londres : Routledge.

Eco, Umberto. 1985. Lector in Fabula. Paris : Grasset et Fasquelle.

Hasan, Ruqaiya. 1984. « The structure of the nursery tale ». In Lorenzo Coveri (dir.), Linguistica Testuale. Rome : Bulzoni, 95-114.

Kael, Pauline. 1993. 5001 Nights at the Movies. New York : M. Boyars.

Keeble, Richard. 1994. The Newspapers Handbook. Londres : Routledge.

McCarthy, Michael \& Ronald Carter. 1994. Language as Discourse. Perspectives for Language Teaching. Londres : Longman.

Simpson, Paul. 1993. Language, Ideology and Point of View. Londres : Routledge.

Swales, John. 1990. Genre Analysis. English in Academic and Research Settings. Cambridge : Cambridge University Press. 


\section{RÉSUMÉS}

L'étude d'un corpus de critiques d'un même film dans les différents quotidiens nationaux britanniques permet de mettre en lumière différents invariants du genre "critique de cinéma ». Nombre de ces constantes sont liées à des facteurs contextuels. Si la présence d'éléments informatifs et évaluatifs sur le film s'explique aisément par la situation des participants (le critique a vu le film, contrairement aux lecteurs), d'autres invariants sont plus inattendus. Ainsi, dans tous les articles se trouvent jeux de mots, mélange de niveaux de langue et passages humoristiques. Cette écriture inventive et divertissante témoigne du besoin des critiques d'attirer des lecteurs autres que ceux intéressés par le cinéma, et s'explique également par la dimension persuasive de ce type de discours, qui vise à convaincre le destinataire d'aller voir ou non un film. Il semble donc que, malgré la diversité des articles du corpus (en termes de type d'humour, de complexité syntaxique des phrases, etc.), il soit possible d'établir des critères de définition du genre « critique de cinéma ».

Thanks to the study of the reviews of the same film in the various British national daily newspapers, different invariant elements of the genre 'film review' can be established. Many of them are linked to contextual factors. The presence of informative and evaluative elements about the film can be easily explained by the situation of the participants - the reviewer has seen the film, whereas the reader has not - although other invariants are more unexpected. Thus, all the articles in the corpus contain puns, a mix of various levels of language, and humorous passages. This creative and entertaining writing stems from the need of film reviewers to attract other readers than those interested in cinema, as well as from the persuasive dimension of this kind of discourse, which aims to convince the addressee to see a film or not. It seems, therefore, that in spite of the diversity within the corpus (in terms of kind of humour, of syntactic complexity, etc.), it is possible to establish criteria to define the genre 'film review'.

\section{INDEX}

Keywords : context, discourse analysis, review

Mots-clés : analyse du discours, contexte, critique, genre, invariant

\section{AUTEUR}

\section{DOROTHÉE BAUD}

Dorothée Baud est professeur agrégée d'anglais, élève en quatrième année à l'École normale supérieure de Cachan et en première année de thèse à l'Université Victor Segalen Bordeaux 2 (EA 2025). Dorothee.Butigieg@ens.fr 